\title{
Expression and clinical significance of the Trop-2 gene in advanced non-small cell lung carcinoma
}

\author{
AIGUI JIANG ${ }^{1}$, XIAOYAN GAO ${ }^{1}$, DEGENG ZHANG $^{2}$, LIXIN ZHANG ${ }^{3}$ and HUIYU LU ${ }^{1}$ \\ Departments of ${ }^{1}$ Respiratory Medicine and ${ }^{2}$ Oncology; ${ }^{3}$ Institute of Clinical Medicine, \\ Taizhou People's Hospital Affiliated to Medical College of The Nantong University, \\ Taizhou, Jiangsu 225300, P.R. China
}

Received January 14, 2013; Accepted May 22, 2013

DOI: $10.3892 / \mathrm{ol} .2013 .1368$

\begin{abstract}
The Trop-2 gene has been examined in various carcinomas and is reported to be significantly associated with prognosis. Little is known with regard to Trop-2 gene expression in advanced non-small cell lung carcinoma (NSCLC). The present study investigated the expression of Trop-2 and its association with the prognosis of advanced NSCLC. The clinical records of 87 patients with advanced NSCLC, consisting of 37 cases of squamous cell carcinoma (SCC) and 50 cases of adenocarcinoma (AdC), together with 17 tumor-adjacent normal tissues, were retrospectively evaluated. Trop-2 expression was measured using an immunohistochemical method and its association with clinicopathological data and prognosis was also evaluated. The expression of Trop-2 was significantly higher in the cancer tissues compared with the tumor-adjacent normal tissues, and significantly higher in SCC compared with AdC ( $\mathrm{P}=0.018)$. In SCC, the overexpression of Trop-2 was only correlated with the histological grade of the tumor $(\mathrm{P}=0.035)$ and no correlation was observed with gender, age, lymph node metastasis, TNM stage or Eastern Cooperative Oncology Group (ECOG) performance status (PS). In AdC, the overexpression of Trop-2 was correlated with the histological grade, lymph node metastasis and TNM stage $(\mathrm{P}=0.01,0.024$ and 0.015 , respectively), while no correlation with gender, age or ECOG-PS was observed. The survival frequency was significantly higher in the Trop-2-negative patients compared with the Trop-2-positive patients [17.25 months (95\% CI, 14.922-19.577) vs. 13.274 months (95\% CI, 11.507-15.041); $\mathrm{P}=0.008]$. The survival time was significantly longer in
\end{abstract}

Correspondence to: Professor Huiyu Lu or Dr Aigui Jiang, Department of Respiratory Medicine, Taizhou People's Hospital Affiliated to Medical College of The Nantong University, 210 Yingchun Road, Taizhou, Jiangsu 225300, P.R. China

E-mail: tzhuxi888@sina.com

E-mail: jiangaigui@126.com

Key words: non-small cell lung carcinoma, trop-2, prognosis, survival the Trop-2-negative AdC patients [17.275 months (95\% CI, 14.575-19.975) vs. 11.469 months (95\% CI, 11.507-15.041); $\mathrm{P}=0.002]$, but not in the SCC patients [17.167 months (95\% CI, 12.428-21.906) vs. 14.647 months (95\% CI, 12.062-17.232); $\mathrm{P}=0.276]$. The multivariate analysis revealed that Trop-2 expression [hazard ratio (HR) 2.381; $\mathrm{P}=0.038$ ], TNM stage (HR, 2.193; $\mathrm{P}=0.03)$ and ECOG-PS (HR, 2.696; $\mathrm{P}=0.007)$ were independent predictors for the survival outcome of patients with AdC. These results suggest that Trop-2 overexpression is closely correlated with an unfavorable prognosis in advanced NSCLC. Trop- 2 is an independent prognostic marker and a potential new therapeutic target in advanced AdC.

\section{Introduction}

Although there has been considerable progress in recent years in the development of anti-cancer therapies, including chemotherapy, radiation therapy and biological targeted therapy, the mortality of patients with advanced non-small cell lung cancer (NSCLC) remains high due to the inability to treat the condition surgically (1). As a consequence, the research into novel prognostic biomarkers and therapeutic target structures in advanced NSCLC remains a focus of attention.

The human trophoblast cell-surface antigen, Trop-2, is a transmembrane glycoprotein originally found to be expressed at high levels on the surface of trophoblastic cells (2). More recent studies have identified Trop-2 to be highly expressed in a number of human epithelial tumors, including colorectal cancer (3), oral squamous cell carcinoma (4) and pancreatic cancer (5), and high expression is often associated with a poor prognosis. By contrast, Trop-2 expression is minimal or absent in normal epithelial tissues. This means that Trop-2 may promote tumor cell proliferation and aggressiveness (6). Trop-2 expression has also been detected in the early stage of NSCLC, but its clinical significance in operative NSCLC remains controversial $(7,8)$. To the best of our knowledge, Trop-2 expression in advanced NSCLC and its association with prognosis has not yet been reported. In the present retrospective study, Trop-2 antigen expression and its correlation with clinicopathological features was evaluated in advanced NSCLC. 


\section{Patients and methods}

Patients. The clinical records of 87 patients (61 males and 26 females; mean age 63.4 years; range, $45-71$ years) with advanced NSCLC who were admitted to the Taizhou People's Hospital (Taizhou, Jiangsu, China) between June 2008 and June 2010 were retrospectively evaluated. In total, 37 cases of squamous-cell carcinoma (SCC), 50 cases of adenocarcinoma $(\mathrm{AdC})$ and 17 tumor-adjacent normal tissue samples were obtained. All cases were confirmed using CT-guided percutaneous or bronchoscopic lung biopsies. The patients were divided into stage IIIb and stage IV tumor groups, according to the TNM system (9). The standard procedure that was used for the inoperable stage IIIb patients was that of sequential chemo-radiation. Platinum-based doublets in two to three cycles were administered prior to irradiation. The platinum doublets were similar to those used for stage IV tumors. Radiation was administered using a linear accelerator $(\geq 6 \mathrm{MeV}$ ) or cobalt- 60 , for a total dose of $50-60 \mathrm{~Gy}$, delivered in 25-30 fractions of 2 Gy/day, 5 days/week. Patients were excluded from the study if they had received prior chemotherapy or radiotherapy, had no definitive histological diagnosis, had a bad performance status (PS; ECOG $\geq 3$ ), had brain tumor metastasis or if they had a disease other than lung cancer that may have affected survival, including cardiac dysfunction, renal insufficiency, liver cirrhosis or concomitant malignancy. This study was approved by the Ethics Committee of Taizhou People's Hospital, Jiangsu, China and was performed according to the Declaration of Helsinki. Written informed consent was obtained from each patient's family.

Immunohistochemistry. Paraffin-embedded tissue blocks were cut into $4-\mu \mathrm{m}$ sections and analyzed immunohistochemically (EliVision ${ }^{\mathrm{TM}}$ Plus IHC kit; Wuhan Boster Biological Engineering Co., Ltd., Wuhan, Hubei, China) for Trop-2 expression (1:50; goat polyclonal antibody; R\&D Systems, Minneapolis, MN, USA). The sections were dewaxed in xylene and rehydrated using graded concentrations of ethanol. The endogenous peroxidase activity was blocked by incubating the sections in 5\% hydrogen peroxide in absolute methanol at room temperature for $10 \mathrm{~min}$. Antigen retrieval was performed in a microwave oven for two cycles of $10 \mathrm{~min}$ each. The primary antibodies were applied for $1 \mathrm{~h}$ at room temperature and the sections were washed three times using 0.05M Tris-buffered saline (TBS, pH 7.2), prior to $50 \mu \mathrm{lg} \mathrm{Ig} / \mathrm{HRP}$ secondary antibody (Wuhan Boster Biological Engineering Co., Ltd.) being added, followed by incubation for $30 \mathrm{~min}$ at room temperature. The sections were washed three times with TBS and the reaction products were visualized with diaminobenzidine (DAB kit; Wuhan Boster Biological Engineering Co., Ltd.). The sections were counterstained with hematoxylin and eosin, dehydrated and evaluated under a light microscope.

Immunohistochemistry scoring. Positive staining for Trop-2 expression was assessed in 10 high-power fields of each tumor by two independent pathologists using light microscopy in a blinded fashion. Trop-2 expression was evaluated for each tissue sample by calculating a total immunostaining score as the product of a proportion and intensity score (5). The proportion score described the estimated fraction of positively-stained tumor cells ( 0 , none; $1, \leq 10 \% ; 2,10-50 \% ; 3,51-80 \%$; and $4, \geq 80 \%)$. The intensity score represented the estimated staining intensity ( 0 , no staining; 1 , weak; 2 , moderate; and 3 , strong). Thus, the total score ranged from $0-12$. The positive and negative expression of Trop-2 were defined as a score of $>4$ and $\leq 4$, respectively.

Follow-up. The patients were followed up from the date of the pathological diagnosis until the date of mortality or the last follow-up at the outpatient department. At the time of the last follow-up, 80 patients $(92 \%)$ had succumbed to the tumor and 7 patients (8\%) were lost to follow-up or succumbed to other causes.

Statistical analysis. The statistical analysis was performed using the SPSS 13.0 software (SPSS, Inc., Chicago, IL, USA). The associations between Trop- 2 immunostaining and the clinicopathological parameters (gender, age, histologic grade, lymph node metastasis, TNM stage and ECOG-PS) were analyzed using $\chi^{2}$ and Fisher's exact tests. Overall survival (OS) was calculated from the date of diagnosis to the date of the last follow up or mortality. The cases of patients that were lost to follow-up or had succumbed from any other cause were defined as censored data for the analysis of survival rates. The survival curves were plotted using the Kaplan-Meier method, and P-values were calculated using the log-rank test. A multivariate analysis was performed using the Cox-proportional hazards model to identify independent prognostic factors. $\mathrm{P} \leq 0.05$ was considered to indicate a statistically significant difference.

\section{Results}

Trop-2 expression in tumor-adjacent normal tissues. Trop-2 expression was absent or infrequent in the tunica mucosa bronchiorum. No positive expression was observed in the alveolar wall. Trop-2 overexpression was detected in $5.9 \%(1 / 17)$ of tumor adjacent normal tissues (Fig. 1).

Trop-2 expression in advanced NSCLC. Staining for Trop- 2 occurred in a diffuse pattern localized mainly in the membrane of the cancer cells, although staining was occasionally identified in the nucleus and cytoplasm (Figs. 2 and 3). Trop-2 overexpression was detected in 52.9\% (46/87) of the tumors. Trop-2 expression was higher in the advanced NSCLC tissues than in the tumor-adjacent normal tissues $(\mathrm{P}=0.000)$, and higher in the SCC cases [67.6\% (25/37)] than in the AdC cases [42.0\% (21/50); $\mathrm{P}=0.018]$

Trop-2 expression associations with clinicopathological variables and prognosis. Trop-2 overexpression in SCC did not differ significantly with regard to patient gender, age, lymph node metastasis, TNM stage or ECOG-PS. However, Trop-2 overexpression was significantly correlated with the histological grade $(\mathrm{P}=0.035$; Table I). Trop-2 overexpression in AdC did not differ significantly with regard to patient gender, age or ECOG-PS. However, Trop-2 overexpression was significantly correlated with the histological grade, lymph 
Table I. Trop-2 expression in association with clinicopathological factors in SCC.

\begin{tabular}{|c|c|c|c|c|c|c|}
\hline \multirow[b]{2}{*}{ Characteristics } & \multirow[b]{2}{*}{ Number } & \multicolumn{2}{|c|}{ Trop-2 overexpression } & \multirow[b]{2}{*}{ Positive rate $(\%)$} & \multirow[b]{2}{*}{$\chi^{2}$} & \multirow[b]{2}{*}{ P-value } \\
\hline & & No & Yes & & & \\
\hline \multicolumn{7}{|l|}{ Gender } \\
\hline Male & 25 & 7 & 18 & 72.0 & 0.6911 & 0.406 \\
\hline Female & 12 & 5 & 7 & 58.3 & & \\
\hline \multicolumn{7}{|l|}{ Age (years) } \\
\hline$\geq 60$ & 19 & 5 & 14 & 73.7 & 0.6668 & 0.414 \\
\hline$<60$ & 18 & 7 & 11 & 61.1 & & \\
\hline \multicolumn{7}{|c|}{ Degree of differentiation } \\
\hline Low-middle & 16 & 2 & 14 & 87.5 & 5.1110 & 0.035 \\
\hline High & 21 & 10 & 11 & 52.4 & & \\
\hline \multicolumn{7}{|c|}{ Lymph node metastasis } \\
\hline No & 10 & 5 & 5 & 50.0 & 1.9299 & 0.165 \\
\hline Yes & 27 & 7 & 20 & 74.1 & & \\
\hline \multicolumn{7}{|l|}{ TNM stage } \\
\hline IIIb & 20 & 8 & 12 & 60.0 & 1.1376 & 0.319 \\
\hline IV & 17 & 4 & 13 & 76.5 & & \\
\hline \multicolumn{7}{|l|}{ PS score } \\
\hline $0-1$ & 14 & 6 & 8 & 57.1 & 1.1169 & 0.291 \\
\hline 2 & 23 & 6 & 17 & 73.9 & & \\
\hline
\end{tabular}

Table II. Trop-2 expression in association with clinicopathological factors in AdC.

\begin{tabular}{|c|c|c|c|c|c|c|}
\hline \multirow[b]{2}{*}{ Characteristics } & \multirow[b]{2}{*}{ Number } & \multicolumn{2}{|c|}{ Trop-2 overexpression } & \multirow[b]{2}{*}{ Positive rate $(\%)$} & \multirow[b]{2}{*}{$\chi^{2}$} & \multirow[b]{2}{*}{ P-value } \\
\hline & & No & Yes & & & \\
\hline \multicolumn{7}{|l|}{ Gender } \\
\hline Male & 36 & 22 & 14 & 38.9 & 0.5109 & 0.475 \\
\hline Female & 14 & 7 & 7 & 50.0 & & \\
\hline \multicolumn{7}{|l|}{ Age (years) } \\
\hline$\geq 60$ & 28 & 17 & 11 & 39.3 & 0.1925 & 0.661 \\
\hline$<60$ & 22 & 12 & 10 & 45.5 & & \\
\hline \multicolumn{7}{|c|}{ Degree of differentiation } \\
\hline Low-middle & 25 & 10 & 15 & 60.0 & 6.6502 & 0.010 \\
\hline High & 25 & 19 & 6 & 24.0 & & \\
\hline \multicolumn{7}{|c|}{ Lymph node metastasis } \\
\hline No & 14 & 12 & 2 & 14.3 & 6.1309 & 0.024 \\
\hline Yes & 36 & 17 & 19 & 52.8 & & \\
\hline \multicolumn{7}{|l|}{ TNM stage } \\
\hline IIIb & 29 & 21 & 8 & 27.6 & 5.8888 & 0.015 \\
\hline IV & 21 & 8 & 13 & 61.9 & & \\
\hline \multicolumn{7}{|l|}{ PS score } \\
\hline $0-1$ & 17 & 12 & 5 & 29.4 & 1.6756 & 0.196 \\
\hline 2 & 33 & 17 & 16 & 48.5 & & \\
\hline
\end{tabular}

AdC, adenocarcinoma; PS, performance status. 


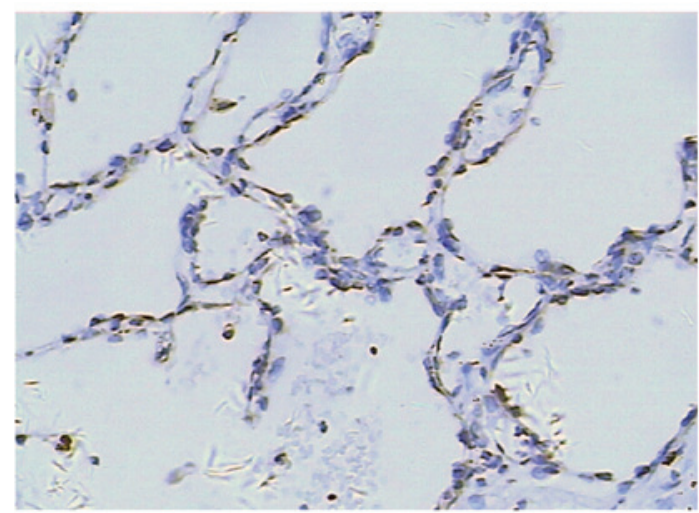

Figure 1. Trop-2 expression in the tumor-adjacent normal lung tissues (hematoxylin \& eosin; x400 magnification).

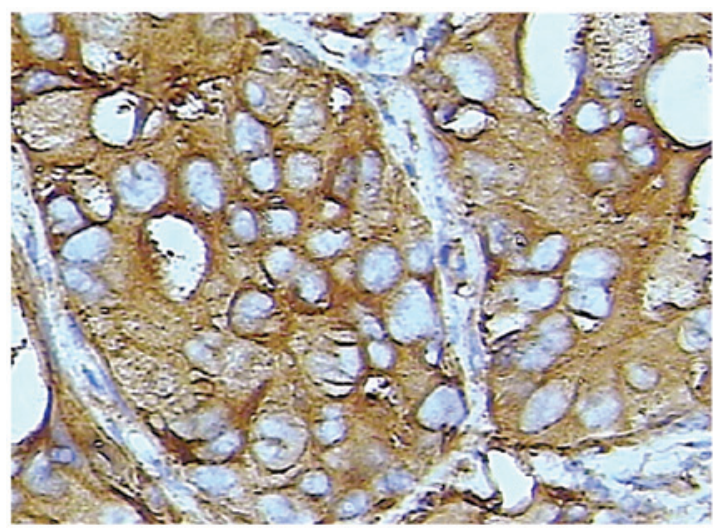

Figure 2. Trop-2 expression in SCC tissues (hematoxylin and eosin; $x 400$ magnification). SCC, squamous-cell carcinoma

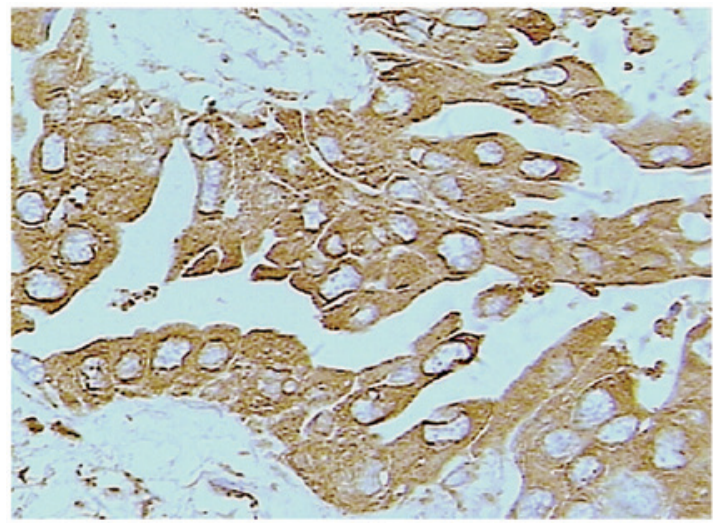

Figure 3. Trop-2 expression in Adc tissues (hematoxylin and eosin; $x 400$ magnification). AdC, adenocarcinoma.

node metastasis and TNM stage $(\mathrm{P}=0.01,0.024$ and 0.015 , respectively; Table II).

The median OS time of all patients was 15.197 months (95\% CI, 13.688-16.706). The survival time was significantly better in the patients with Trop-2-negative expression than those with Trop-2-positive expression [17.25 months (95\% CI, 14.922-19.577) vs. 13.274 months (95\% CI, 11.507-15.041); $\mathrm{P}=0.008]$. The survival time was significantly longer in

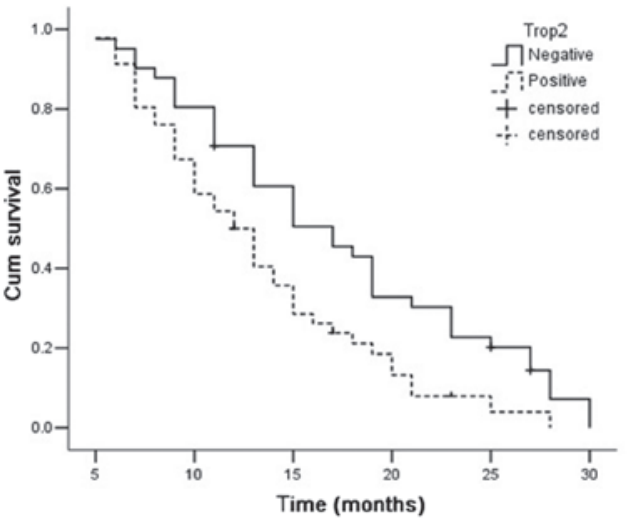

Figure 4. Kaplan-Meier curves in advanced NSCLC patients positive and negative for Trop-2 (Log Rank, $\left.\chi^{2}=7.094, \mathrm{P}=0.008\right)$. NSCLC, non-small cell lung carcinoma; Cum, cumulative.

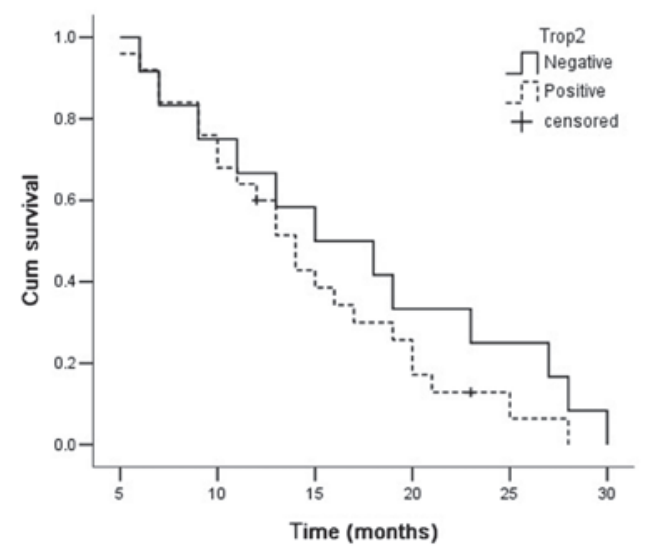

Figure 5. Kaplan-Meier curves in advanced squamour cell carcinoma (SCC) positive and negative for Trop- $2\left(\log \operatorname{Rank}, \chi^{2}=1.186, \mathrm{P}=0.276\right)$. Cum, cumulative.

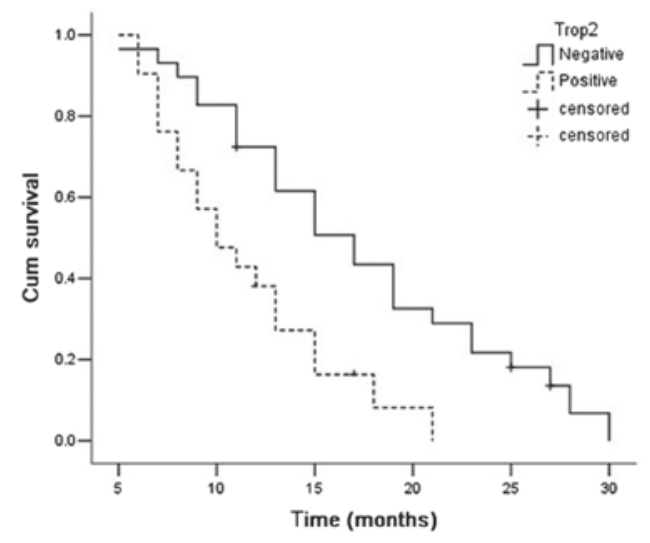

Figure 6. Kaplan-Meier curves in advanced adenocarcinoma (AdC) patients positive and negative for Trop- $2\left(\log \operatorname{Rank}, \chi^{2}=9.611, \mathrm{P}=0.002\right)$. Cum, cumulative.

the Trop-2-negative AdC patients [17.275 months (95\% CI , 14.575-19.975) vs. 11.469 months (95\% CI, 11.507-15.041); $\mathrm{P}=0.002]$, but not in the SCC patients [17.167 months (95\% CI, 12.428-21.906) vs. 14.647 months (95\% CI, 12.062-17.232); $\mathrm{P}=0.276$; Figs. 4-6]. 
Table III. Multivariate analysis of survival in advanced pulmonary adenocarcinoma.

\begin{tabular}{|c|c|c|c|c|c|}
\hline Parameter & Regression co-efficient & Standard error & Wald & $\mathrm{HR}(95 \% \mathrm{CI})$ & P-value \\
\hline Trop-2 overexpression & 0.868 & 0.417 & 4.325 & $2.381(1.051-5.394)$ & 0.038 \\
\hline Age ( $\geq 60$ vs. $<60)$ & -0.154 & 0.433 & 0.126 & $0.857(0.367-2.004)$ & 0.722 \\
\hline Gender (male vs. female) & 0.285 & 0.410 & 0.484 & $1.330(0.595-2.972)$ & 0.487 \\
\hline TNM stage (IIIb vs. IV) & 0.785 & 0.361 & 4.727 & $2.193(1.080-4.453)$ & 0.030 \\
\hline $\begin{array}{l}\text { Degree of differentiation } \\
\text { (Low-middle vs. high) }\end{array}$ & -0.362 & 0.429 & 0.714 & $0.696(0.300-1.614)$ & 0.398 \\
\hline $\begin{array}{l}\text { Lymph node metastasis } \\
\text { (Yes vs. no) }\end{array}$ & 0.477 & 0.388 & 1.510 & $1.611(0.753-3.448)$ & 0.219 \\
\hline PS score (0-1 vs. 2$)$ & 0.992 & 0.368 & 7.254 & $2.696(1.310-5.549)$ & 0.007 \\
\hline
\end{tabular}

CI, confidence interval; PS, performance status; HR, hazard ratio.

In the univariate survival tests of AdC that were performed using clinicopathological factors, the statistically significant parameters, other than Trop-2 expression (HR, 2.606; $\mathrm{P}=0.004$ ) were lymph node metastasis (HR, 2.258; $\mathrm{P}=0.011)$, TNM stage $(\mathrm{HR}, 2.478 ; \mathrm{P}=0.005)$ and ECOG-PS (HR, 2.586; $\mathrm{P}=0.005)$. Other variables, including age, gender and tumor differentiation, were not associated with a better survival outcome. In the multivariate survival tests, Trop-2 expression (HR, 2.381; $\mathrm{P}=0.038)$, TNM stage (HR, 2.193; $\mathrm{P}=0.03)$ and ECOG-PS $(\mathrm{HR}, 2.696 ; \mathrm{P}=0.007)$ were identified as independent prognostic markers in advanced AdC (Table III).

\section{Discussion}

The Trop-2 protein (also termed GA733-1, M1S1 and EGP-1), is a human trophoblast cell-surface antigen encoded by the TACSTD2 gene of human chromosome 1p32 (10). The TACSTD2 gene lacks introns and is formed by exon shuffling and retroposition of the TACSTD1 gene via an mRNA intermediate. TACSTD2 encodes a $35 \mathrm{kDa}$, type 1 transmembrane protein, which contains 323 amino acids and a single transmembrane domain. Trop-2 is a calcium channel protein that is associated with the regulation of intracellular calcium concentration (11). Moreover, Trop-2 plays a significant role in the regulation of tumor proliferation by increasing the stability of cyclin D1 or activating the signal pathway of ERKl-MAPK $(12,13)$.

Early studies identified Trop-2 gene mutations to be associated with Gelatinous Drop-like Corneal Dystrophy (GDLD), a rare autosomal recessive genetic disease which leads to severe vision disorders and even blindness (14-16). More recently, studies have observed that Trop-2 is highly expressed in a number of human epithelial tumors compared with the restricted expression found in normal tissues, and that it is also associated with poor overall patient survival (3-5). These previous studies have revealed that Trop-2 may promote tumor proliferation, aggressiveness and metastasis (6). Trop-2 expression has also been detected in the early stage of NSCLC, but its clinical significance in operative NSCLC remains controversial $(7,8)$. Kobayashi et al $(7)$ revealed that 87 of 130 patients $(67 \%)$ with small-sized pulmonary $\mathrm{AdC}$ ( $<2 \mathrm{~cm}$ diameter) were immunopositive for Trop-2 expression and therefore associated with a poor OS. The multivariate analysis showed that Trop-2 overexpression was an independent, unfavorable prognostic marker in AdC and NSCLC. Pak et al (8) reported that 64 of 164 patients (39\%) with stage II or III NSCLC were immunopositive for Trop-2 expression. The Trop-2 expression in patients with AdC [23/100 (23\%)] was significantly lower than that in the SCC patients [41/64 (64\%)]. Trop-2 overexpression showed a better $\mathrm{OS}$ in the $\mathrm{AdC}$ patients. The inconsistent results between the two studies suggest that the biological role of Trop-2 may vary between early and advanced pulmonary AdC.

To the best of our knowledge, there are no studies with regard to the correlation between Trop-2 expression and advanced NSCLC in the English-language literature. The present study detected that Trop-2 expression occurred in the membrane of lung cancer cells and occasionally in the nucleus and cytoplasm. Trop-2 expression was higher in advanced NSCLC than in the tumor-adjacent normal tissues. These results revealed that the Trop-2 gene may be associated with tumorigenesis and that the progression of advanced NSCLC. Trop-2 expression in the present study was significantly higher in NSCLC than in the tumor-adjacent normal tissues. Additionally, Trop-2 was significantly overexpressed in SCC compared with $\mathrm{AdC}$, as observed in a previous study (8). The present study also detected that Trop-2 overexpression in SCC was only correlated with the histological grade of the tumor. However, Trop-2 expression in AdC was correlated with the histological grade, lymph node metastasis and TNM stage. The multivariate analysis showed that Trop- 2 is an independent prognostic marker in advanced pulmonary AdC and that it may play a more significant role in the pathogenesis of this disease.

In conclusion, Trop-2 is closely correlated with unfavorable prognostic factors in advanced NSCLC. Trop- 2 is also an independent prognostic marker in advanced AdC. The present results further indicate that Trop-2 may be a potential new therapeutic target for advanced AdC. However, due to the limitations inherent in retrospective analyses, the prognostic 
value of Trop-2 overexpression requires further validation in larger prospective studies.

\section{References}

1. Jemal A, Bray F, Center MM, Ferlay J, Ward E and Forman D: Global cancer statistics. CA Cancer J Clin 61: 69-90, 2011.

2. Jiang HF and Bo JJ: Trophoblastic cell surface protein 2 and tumor. J Int Oncol 39: 96-98, 2012 (In Chinese).

3. Ohmachi T, Tanaka F, Mimori K, Inoue H, Yanaga $\mathrm{K}$ and Mori M: Clinical significance of TROP2 expression in colorectal cancer. Clin Cancer Res 12: 3057-3063, 2006.

4. Fong D, Spizzo G, Gostner JM, et al: TROP2: a novel prognostic marker in squamous cell carcinoma of the oral cavity. Mod Pathol 21: 186-191, 2008.

5. Fong D, Moser P, Krammel C, et al: High expression of TROP2 correlates with poor prognosis in pancreatic cancer. Br J Cancer 99: 1290-1295, 2008.

6. Wang J, Day R, Dong Y, Weintraub SJ and Michel L: Identification of Trop-2 as an oncogene and an attractive therapeutic target in colon cancers. Mol Cancer Ther 7: 280-285, 2008.

7. Kobayashi H, Minami Y, Anami Y, et al; IASLC International Staging Committee; Cancer Research and Biostatistics; Observers to the Committee; Participating Institutions: Expression of the GA733 gene family and its relationship to prognosis in pulmonary adenocarcinoma. Virchows Arch 457: 69-76, 2010.

8. Pak MG, Shin DH, Lee $\mathrm{CH}$ and Lee MK: Significance of EpCAM and TROP2 expression in non-small cell lung cancer. World J Surg Oncol 10: 53, 2012.
9. Groome PA, Bolejack V, Crowley JJ, et al; IASLC International Staging Committee; Cancer Research and Biostatistics; Observers to the Committee; Participating Institutions: The IASLC Lung Cancer Staging Project: validation of the proposals for revision of the T, N, and $\mathrm{M}$ descriptors and consequent stage groupings in the forthcoming (seventh) edition of the TNM classification of malignant tumors. J Thorac Oncol 2: 694-705, 2007.

10. Calabrese G, Crescenzi C, Morizio E, Palka G, Guerra E and Alberti S: Assignment of TACSTD1 (alias TROP1, M4S1) to human chromosome 2 p21 and refinement of mapping of TACSTD2 (alias TROP2, M1S1) to human chromosome 1p32 by in situ hybridization. Cytogenet Cell Genet 92: 164-165, 2001.

11. Taylor JT, Zeng XB, Pottle JE, et al: Calcium signaling and T-type calcium channels in cancer cell cycling. World J Gastroenterol 14: 4984-4991, 2008.

12. Nelsen CJ, Kuriyama R, Hirsch B, et al: Short term cyclin D1 overexpression induces centrosome amplification, mitotic spindle abnormalities, and aneuploidy. J Biol Chem 280: 768-776, 2005.

13. Cubas R, Zhang S, Li M, Chen C and Yao Q: Trop2 expression contributes to tumor pathogenesis by activating the ERK MAPK pathway. Mol Cancer 9: 253, 2010.

14. Zhang B, Yao YF and Zhou P: Two novel mutations identified in two Chinese gelatinous drop-like corneal dystrophy families. Mol Vis 13: 988-992, 2007.

15. Markoff A, Bogdanova N, Uhlig CE, Groppe M, Horst J and Kennerknecht I: A novel TACSTD2 gene mutation in a Turkish family with a gelatinous drop-like corneal dystrophy. Mol Vis 12: 1473-1476, 2006.

16. Alavi A, Elahi E, Tehrani MH, et al: Four mutations (three novel, one founder) in TACSTD2 among Iranian GDLD patients. Invest Ophthalmol Vis Sci 48: 4490-4497, 2007. 